\title{
Spare parts support model with different repair time distribution
}

$$
\text { ZHOU Liang }{ }^{1, a} \text {, LI Qing- min }{ }^{2, b} \text {, Li Hua }{ }^{1} \text {, PENG Yin-wu }{ }^{1}
$$

${ }^{1}$ Department of weapon Engineering, Naval University of Engineering, Wuhan430033,china

${ }^{2}$ Office of Research \& Department, Naval University of Engineering, Wuhan 430033, China

aE-mail: zh201314l@163.com ${ }^{\text {b} E-m a i l: ~ l i c h e n g 001 @ h o t m a i l . c o m ~}$

\section{Keywords: Spare parts; repair time distribution; simulation; availability}

\begin{abstract}
Spare parts are one of the important supporting resources of equipment. According to the different distribution of maintenance time in spare parts support model, the model of spare parts support models with exponential distribution and constant distribution are established. According to the maintenance and supply of spare parts, the simulation model is established based on discrete event and Monte Carlo simulation. The equipment availability of different spares support model under different repair probability are calculated and compared. The results show that: when the spare parts repair probability is less than 1, we can use the constant distribution model to replace exponential distribution model, and this can greatly improve the computational efficiency.
\end{abstract}

\section{Introduction}

Spare parts are one of the important resources for the equipment work continually. When the equipment breaks down, the repair teams will replace the failed parts with spare parts. The Repair time of Spare parts obeys constant distribution or exponential distribution.

For multi-level system, the literature [1] studied the optimal allocation of spare parts for each site. In some system, the number of spare parts is limited by space and the weight which can bear [2].In the literature [3], the model of the spare parts lateral transfer at the same level was established. The spare parts replacement policy of all above is $(s-1, s)$.In fact, for some redundant systems, we will not replace the fault parts until the redundant systems cannot work normally [4]. In the literature [5] and [6], the model of two level spare parts support is established. According Dynamic palm theorem, the supporting model of spare parts is established based on the time-varying availability ${ }^{[8-9]}$. Based on non stationary random process, a model of two level spare parts support is established ${ }^{[10]}$. Taking the single site as the research object, the model of spare parts support is discussed when the repair time obey constant distribution and exponential distribution.

When the equipment breaks down, the repair teams will replace the failed parts with spare parts. If the repair teams can repair the failed parts, the repaired parts will send to warehouse. If the repair team cannot repair the LRU, it will wait to be supplied until the task finished. If the warehouse has not LRU, the failed parts will wait replace until the warehouse has LRU. The maintenance and supply of spare parts is shown in Figure1.

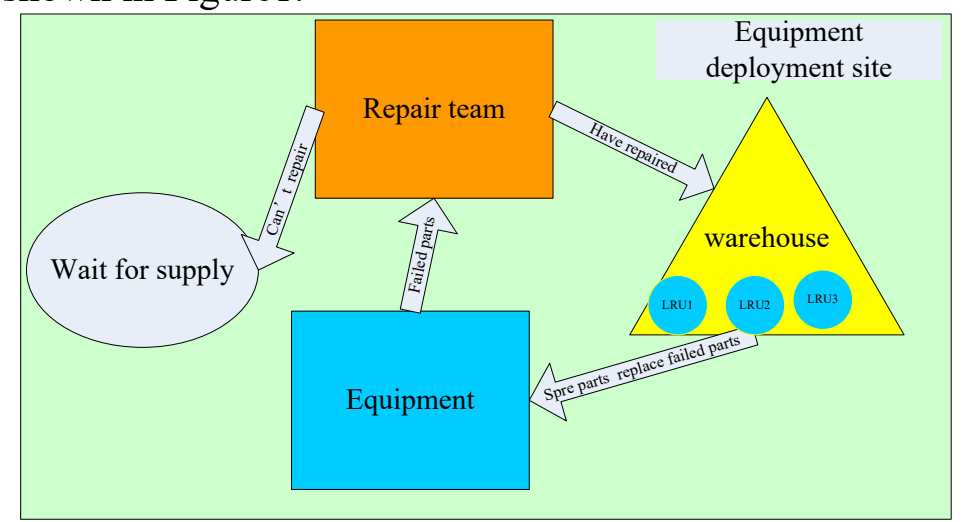

Fig.1 spare parts maintenance and supply process 


\section{Variable Definitions}

$T$ : The task time

$M T B F_{l}$ : The storage life of LRU

$E B O_{l}$ : The expectation shortage number of LRU.

$R P_{l}$ : The repairing number of LRU.

$N R P_{l}$ : The unrepairable number of LRU.

$r_{l}$ : The repairable Probability of LRU.

$M T R_{l}$ : LRU maintenance time.

$S_{l}$ : The spare parts Number of LRU.

\section{Availability Modeling}

At time $t$, the number of maintenance at deployment site can divide into two parts: The number of LRU is repairing; the number of LRU cannot be repaired.

\section{Demand Rate of LRU}

At $t$ time, we can calculate demand number of LRU. If assume the demand number is $N e_{j l}(t)$, we can get:

$$
N e_{j l}(t)=\frac{K_{l}}{M T B F_{s l}}
$$

\section{Exponential Distribution of Repairing Time}

If the LRU failed, they will replace the failed LRU with good LRU. The repair teams judges the failed LRU whether can repair. According to the dynamic palm theorem ${ }^{[1]}$, the expected number of LRU which is repairing is poison distribution.

$$
E\left[R P_{l}(t)\right]=\int_{1}^{t} N e_{l}(s) g_{l} g P K_{l}(s, t) d s
$$

$P K_{l}(r, t)$ express the probability of LRU start to repair at $r$ time, and it have not repair well at $t$ time. The $P K_{l}(r, t)$ is

$$
P K_{l}(r, t)=1-e^{-\frac{t-r}{M R T_{l}}}
$$

\section{Constant Distribution of Repairing Time}

If the LRU failed, they will replace the failed LRU with good LRU. The repair teams judges the failed LRU whether can repair. At time $t$, the expected number of LRU is the formula (4).

$$
E\left[R P_{l}(t)\right]=\left\{\begin{array}{cc}
N e_{l}(t) g M R T_{l} & t \geq M R T_{l} \\
N e_{l}(t) \mathfrak{g} & t \leq M R T_{l}
\end{array}\right.
$$

\section{The number of Maintenance Supply}

(1) Non repairable number

The expected number of LRU which cannot repair well is also poison distribution.

$$
E\left(N R P_{k l}(t)\right)=\int_{1}^{t} N e_{l}(s) g\left(1-r_{l}\right) d s
$$

(2)The number of maintenance and transportation supply

According to the formula (3) and formula (4), we can get the number of maintenance supply which is also poison distribution.

$$
E\left[D I_{k l}(t)\right]=E\left[N R P_{k l}(t)\right]+E\left[R P_{k l}(t)\right]
$$


On the basis of Inventory balance formula from shbrooke ${ }^{[1]}$, the expectation short number of LRU is poison distribution.

$$
E B O_{k l}(t)=\sum_{x=S_{k l}+1}^{\infty}\left(x-S_{k l}\right) \frac{E\left[D I_{k l}(t)\right]^{x}}{x !} \mathrm{g} e^{-E\left[D I_{k l}(t)\right]}
$$

\section{Simulation Modeling}

Using discrete event scheduling and Monte Carlo method, the simulation model is built through MATLAB. Using $t_{f a i l}$ to show the failure time of LRU, $t_{\text {waittime }}$ show the wait time of the LRU. The simulation of spare parts maintenance and supply process is shown in fig2.

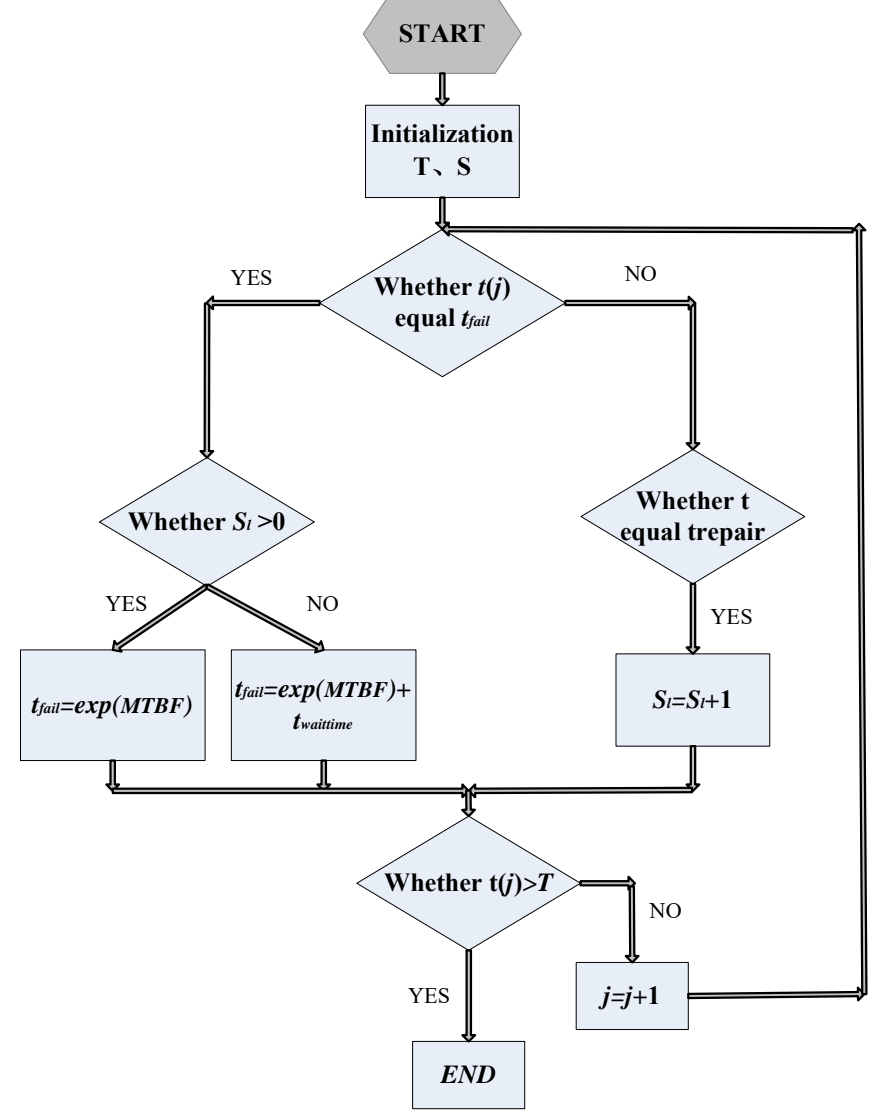

Fig.2 The simulation process

\section{Example}

The reliability information of the components is shown in the table1.

Table1 Reliability information of the components

\begin{tabular}{|c|c|c|}
\hline MTBF & MRT & $\mathrm{S}_{1}$ \\
\hline 1000 & 500 & 1 \\
\hline
\end{tabular}

According to the maintenance time's probability distribution of the spare parts, the analytical model and the simulation model are used to calculate the equipment availability. When the maintenance probability of failed parts is: $1,0.8,0.6,0.4,0.2,0$, the equipment availability changes with time as shown in figure3. 


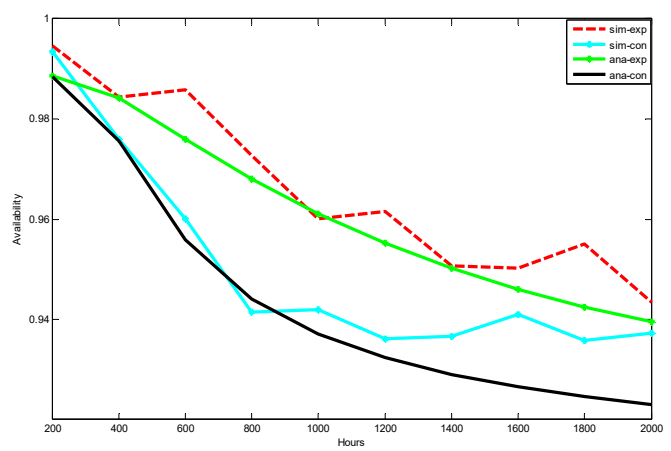

(a) $\mathrm{P}=1$

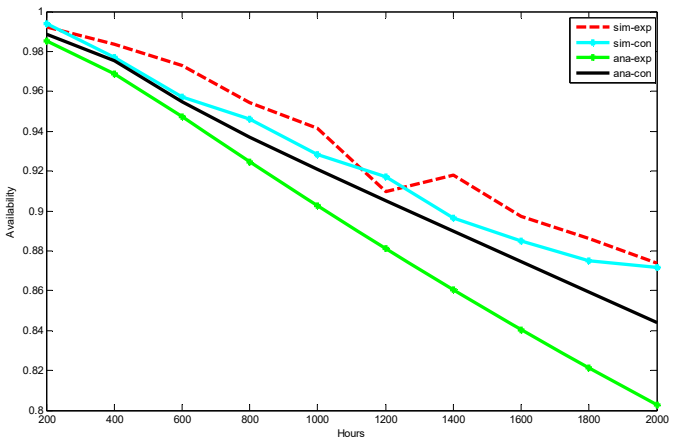

(c) $\mathrm{P}=0.6$

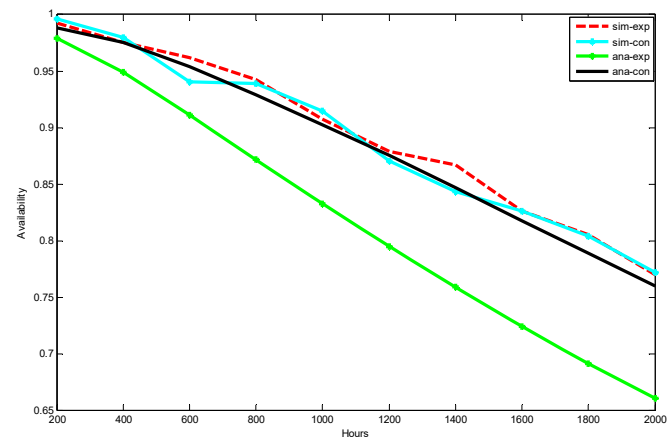

(e) $\mathrm{P}=0.2$

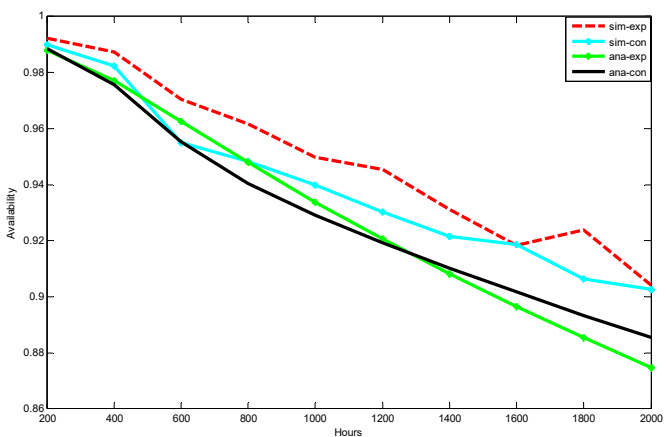

(b) $\mathrm{P}=0.8$

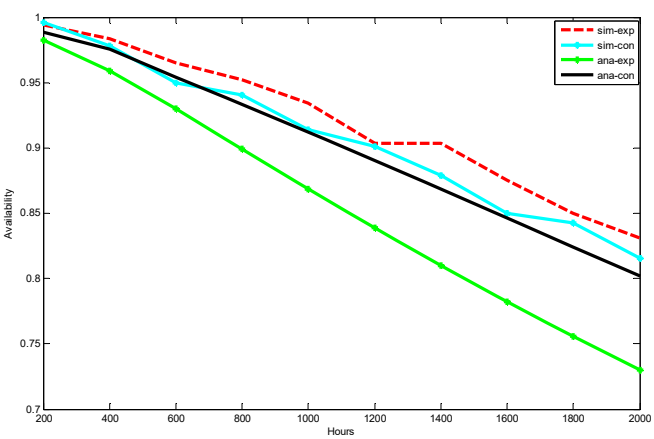

(d) $\mathrm{P}=0.4$

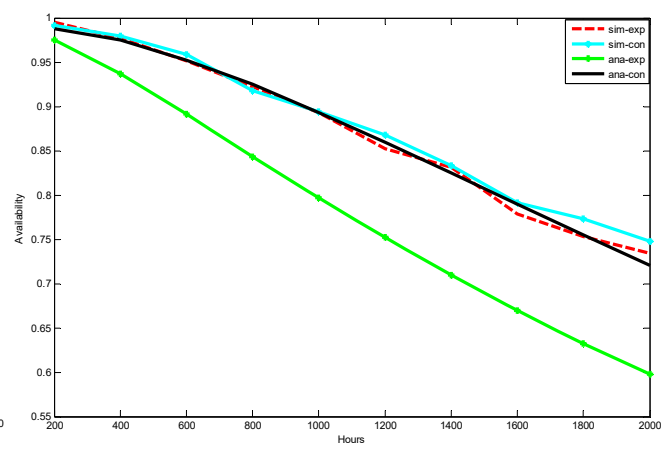

(f) $\mathrm{P}=0$

Fig. 3 the availability of equipment under different maintenance probability

Analysis in Figure 3 can be learned: When the system is fully repairable, as shown in Figure 3 (a), the equipment availability is close to the constant simulation curve when the repair time is constant, and the equipment availability is close to the exponential simulation curve when the repair time is exponential. With the declining probability of the components to be repaired, as shown in Figure 3 (b)-3(f), the simulation curve with constant distribution and the simulation curve of exponential distribution are closer, and the equipment availability and the simulation curve are more close to the time when the repair time is constant.

\section{Conclusion}

In this paper, the model of spare parts support is established when the maintenance time follows the exponential distribution and the constant distribution, and a simulation model for the maintenance and supply of spare parts is established. From the case, we can get the following conclusion: when the spare parts repair probability is less than 1, we can use the constant distribution model to replace exponential distribution model, and this can greatly improve the computational efficiency.

\section{References}

[1]Sherbrook C C. Optimal Inventory Modeling of Systems: Multi-Echelon Techniques (Second 
Edition)[M]. Boston: Artech House, 2004.

[2] RUAN Min-zhi,LI Qing-min,PENG Ying-wu. Modeling and optimization for repair level of ship borne equipment under multi-constraints[J].Systems Engineering and Electronics,2012,5(34).

[3] Lee H L. Modeling emergency lateral transshipments in inventory systems[J]. Management Science, 1987,33:1302-1316.

[4]Ruan Minzhi, Luo Yi,Li Hua. Configuration model of partial repairable spares under batch orderi ng policy based on inventory state[J],Chinese Journal of Aeronautics, 2014, 27(3): 558-567.

[5]Yoon K B, Sohn S Y. Finding the Optimal CSP Inventory Level for Multi-Echelon System in Air Force Using Random Effects Regression Model[J]. European Journal of Operational Research, 2007, 180(3): 1076-1085.

[6]Elisa A, Matthieu H. On two-echelon inventory systems with Poisson demand and lost sales[J]. European Journal of Operational Research, 2014(235): 334-338.

[7]Lau H C, Song H, See T C, et al. Evaluation of time-varying availability in multi echelon spare parts systems with passivation[J]. European Journal of Operational Research. 2006, 170(1): 91-105.

[8] Wang rui,LEI Hong-Wei,PENG Yin-Wu.Optimization of Warship spare parts in the scenario of wartime Mission[J]. Journal of Shanghai Jiao Tong university.2013,47(3),398-403.

[9] Wang rui, PENG Yin-Wu,Ruan Min-zhi.Optimization of repairable spare parts based on combat unit mission success[J]. Journal of Beijing university of Aeronautics and Astronautics.2013,47(3),398-403.

[10]Liu Yong,Wu Chang,LI Yang. Evaluation Model of Equipment Time-varying Availability in Two-echelon Spare Support System.ACTA ARMAMENTARII,2010,2(31),253-256. 